\title{
Analysis of Flame Retardancy in Polymer Blends by Synchrotron X-ray K-edge Tomography and Interferometric Phase Contrast Movies
}

Mutairu B. Olatinwo ${ }^{1}$, Kyungmin $\mathrm{Ham}^{2}$, Jonathan McCarney ${ }^{3}$, Shashidhara Marathe ${ }^{4+}$, Jinghua $\mathrm{Ge}^{5}$, Gerry Knapp ${ }^{6}$, and Leslie G. Butler ${ }^{1}$ *

${ }^{1}$ Department of Chemistry, Louisiana State University, 232 Choppin Hall, Baton Rouge, LA 70803, USA

${ }^{2}$ Center for Advanced Microstructures \& Devices, Louisiana State University, 698o Jefferson Highway, Baton Rouge, LA 70806, USA

${ }^{3}$ Albemarle Corporation, Process Development Center, P. O. Box 341, Baton Rouge, LA 70821, USA

${ }^{4}$ Advanced Photon Source, Argonne National Laboratory, Building 401, 9700 S. Cass Avenue, Argonne, IL 60439, USA

${ }^{5}$ Center for Computation and Technology, Louisiana State University, 340 E Parker Blvd, Baton

Rouge, LA 70808, USA

${ }^{6}$ Department of Mechanical and Industrial Engineering, Louisiana State University, Baton Rouge, LA 70803, USA

${ }^{+}$Division of Science, Diamond Light Source Ltd., Didcot, Oxfordshire, United Kingdom

\section{Table of Contents for Supplementary Material}

\section{Optical photos of UL 94 cut samples}

1.1 Figure S1: An optical image showing char layers and various states of pristine interiors of

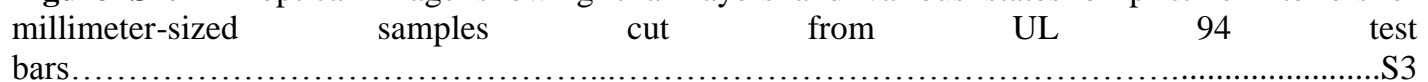

bars.

\section{APS single-shot interferometry}

2.1 Figure S2: An optical image of sample A, only $4 \mathrm{wt} \% \mathrm{Sb}_{2} \mathrm{O}_{3}$ formulation and taken from UL-94 test burn bar show a char layer and a nearly pristine interior.

2.2 Figure S3: An optical image of sample B', having only $12 \mathrm{wt} \%$ BFR formulation and taken from UL-94 test burn bar show a char layer and a nearly pristine

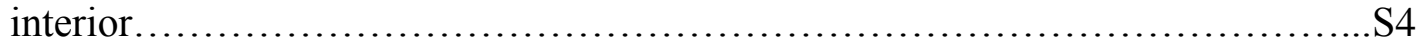

2.3 Figure S4: An optical image of sample C', having $12 \mathrm{wt} \%$ BFR and insufficient $\mathrm{Sb}_{2} \mathrm{O}_{3}$ formulation and taken from UL-94 test burn bar show a char layer and a nearly pristine interior.

2.4 Figure S5: An optical image of sample $\mathrm{D}$, having the correct BFR and $\mathrm{Sb}_{2} \mathrm{O}_{3}$ formulation and taken from UL-94 test burn bar show a char layer and a nearly

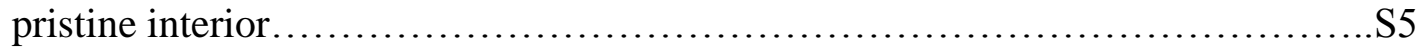

2.5 Figure S6: The autoscaling inherent in single X-ray energy imaging, as for these orthoslices from single-shot interferometry, can lead to misunderstanding of chemical distributions

3. K-edge Absorption Tomography 
3.1 Fig S7: Stacked slice at $12 \mathrm{keV}$, below the $\mathrm{Br}$ edge of sample $\mathrm{A} 1, \mathrm{C} 1$ and

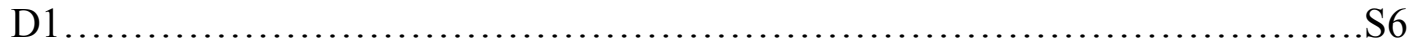

3.2 Figure S8: Stacked slice of sample A1, C1, and D1, D2 of maximum intensity at 29 $\mathrm{keV}$

3.3 Figure S9: Volume renderings of maximum intensity of burnt samples A1, B1 and $\mathrm{C} 1$ S7

3.4 Table S1: BFR Vol\% concentration of samples B1, C1, D1 and D3 with mean, standard deviation and maximum values

3.5 Figure S10: Histogram of $\mathrm{Sb}_{2} \mathrm{O}_{3}$ concentration in vol \% of sample $\mathrm{A} 1, \mathrm{C} 1, \mathrm{D} 1$ and D3. At a threshold of $1.4 \mathrm{vol} \%$, fresh/unburnt sample D3 has a fraction of 8.91 in lumps....

3.6 Table S2: $\mathrm{Sb}_{2} \mathrm{O}_{3}$ Vol\% concentration of samples A1, C1, D1 and D3 with mean, standard deviation and maximum values S8

3.7 Figure S11: Selected representative slice of 15 and $32 \mathrm{keV}$ after Br- and Sb K-edge out of 6 datasets at different energies were used in least squares-fitting method for concentration calculation ( $\mathrm{a}$ and $\mathrm{b}$ in absorption value); $\mathrm{c}$ and $\mathrm{d}$ are BFR and $\mathrm{Sb}_{2} \mathrm{O}_{3}$ concentration in vol\% respectively. This is burnt sample D2 ...................S

3.8 Fig S12: The orthoslice, volume rendering and line probe (radius $=10$ ) of unburnt sample D3 show the blending and good homogeneity of the flame retarded polymer sample.

3.9 Figure S13: A 2D orthoslice (a) with a line probe (radius $=10$ ) to generate graph (c); 3D volume rendering (b) are displayed to present concentration gradient across the char layer of burnt sample D1. It represents low concentration in the char layer for both BFR and $\mathrm{Sb}_{2} \mathrm{O}_{3}$..... S11

3.10 Figure S14: A 2D orthoslice (a) with a line probe (radius $=10$ ) to generate graph (c); 3D volume rendering (b) are displayed to present concentration gradient across the char layer of burnt sample D2. It represents low concentration in the char layer for both BFR and $\mathrm{Sb}_{2} \mathrm{O}_{3}$.... S12

3.11 Figure S15: Volume renderings for the line probes (radius $=10$ ) for comparing BFR concentration in vol\% from the air through the char layer and into the interior for burnt $\mathrm{C} 1$, burnt B1, burnt D1, burnt D2, and pristine D3. The $\mathrm{X}$-axes of the burnt samples are mutually registered based on the distance for the $1.5 \mathrm{vol} \%$ concentration.

3.12 Figure S16: Line probes (radius = 10) for comparing $\mathrm{Sb}_{2} \mathrm{O}_{3}$ concentration in vol\% from the air through the char layer and into the interior for burnt $\mathrm{C} 1$, burnt

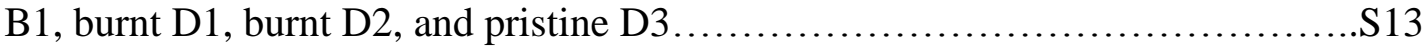

\section{Single-Shot Interferometry Movies}

4.1 Figure S17: Phase wraps are shown in selected DPC slices of interferometry movies, 1.3 pixel size at $22 \mathrm{keV}$.

Dropbox links to movies in Quicktime format, about 30MB each, zipped into one file. https://www.dropbox.com/s/4dlosjihkra7tnf/FR_movies\%20for\%20samples\%20C\%20and $\% 20$ D.zip? $\mathrm{dl}=0$

D_Expt21_abs.mov, D_Expt21_PH.mov, D_Expt21_DI.mov. 
C_Expt20_abs.mov, C_Expt20_PH.mov, C_Expt20_DI.mov.

UL 94 Test Samples:

Fresh polymer test bars used for standard UL 94 vertical burn method have dimension of $12.5 \times 1.3 \times 0.32$ cubic centimeter. Due to prior detector camera limitations, various cuts of $10 \mathrm{~mm}$ height and $1.2 \mathrm{~mm}$ in diameter were made from the burnt test bars for imaging in $\mathrm{X}$-ray synchrotron tomography and phase contrast interferometry.

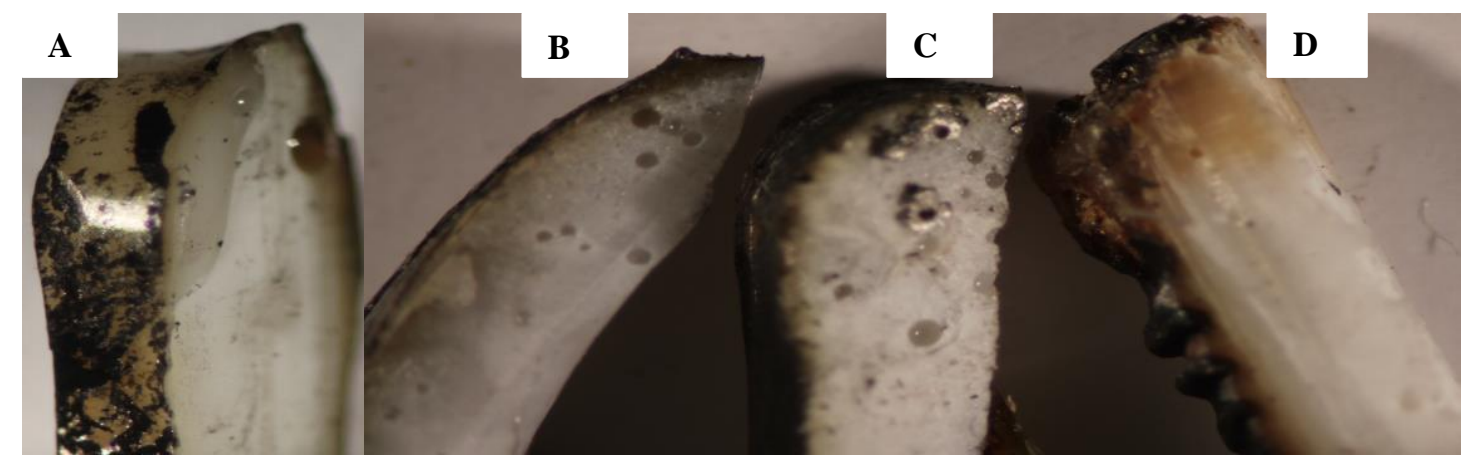

Figure S1: An optical image showing char layers and various states of pristine interiors of millimeter-sized samples cut from UL 94 test bars. Sample D, having the correct BFR and $\mathrm{Sb}_{2} \mathrm{O}_{3}$ formulation, appears to have the thickest char layer. Sample A, having only $\mathrm{Sb}_{2} \mathrm{O}_{3}$, shows the most pristine interior; this is attributed to fast burn-off of exterior layers of the sample. Sample A does not show gas bubbles; hence the polystyrene is not contributing to gas bubble growth. The thickness of the char layer in samples $\mathrm{B}$ and $\mathrm{C}$ are low and they do appear different from sample D.

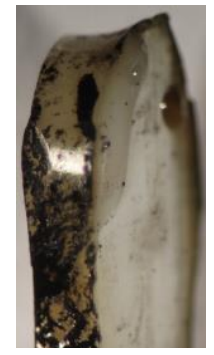

A': photo

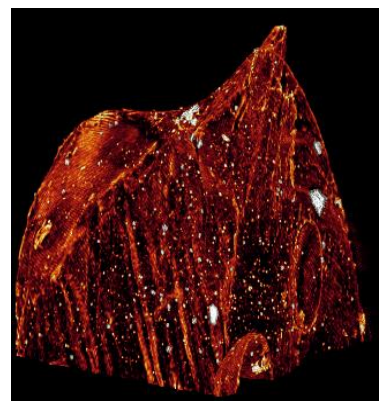

Absorption volume

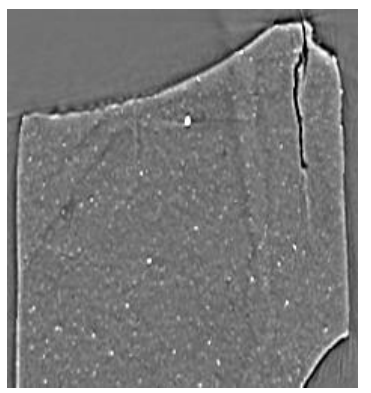

Absorption slice
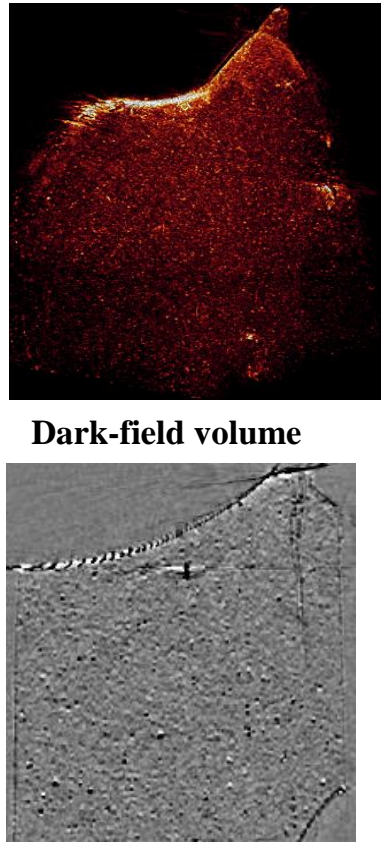

Dark-field slice

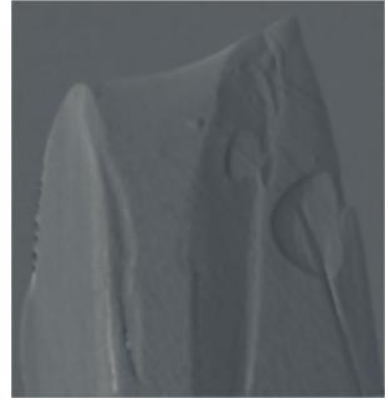

DPC projection horizontal

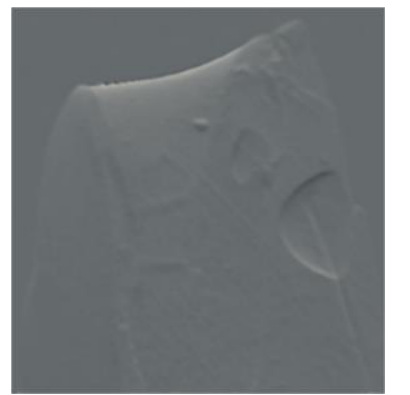

DPC projection vertical

Figure S2: An optical image of sample A, only $4 \mathrm{wt} \% \mathrm{Sb}_{2} \mathrm{O}_{3}$ formulation and taken from UL-94 test burn bar show a char layer and a nearly pristine interior. Tomography volumes 
and orthoslices of absorption, dark-field and horizontal with vertical projections of DPC show absence of the char layer as well as gas-bubbles by effects of heating.

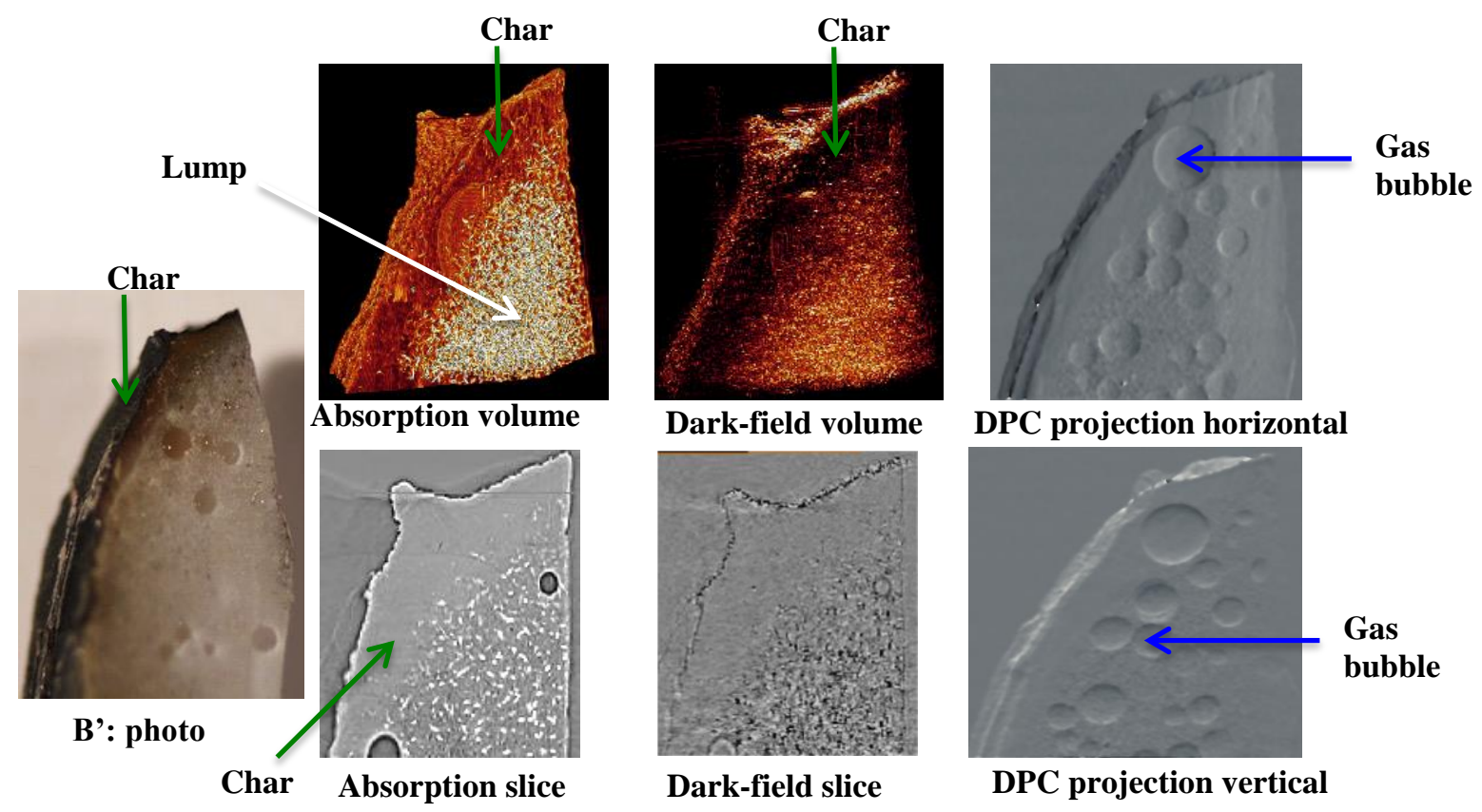

Figure S3: An optical image of sample B', having only $12 \mathrm{wt} \%$ BFR formulation and taken from UL-94 test burn bar show a char layer and a nearly pristine interior. Tomography volumes and orthoslices of absorption, dark-field and horizontal with vertical projections of DPC show the char layer as well as an interior region that shows effects of heating, with possible dissolution of BFR lumps and the formation of gas-bubbles.

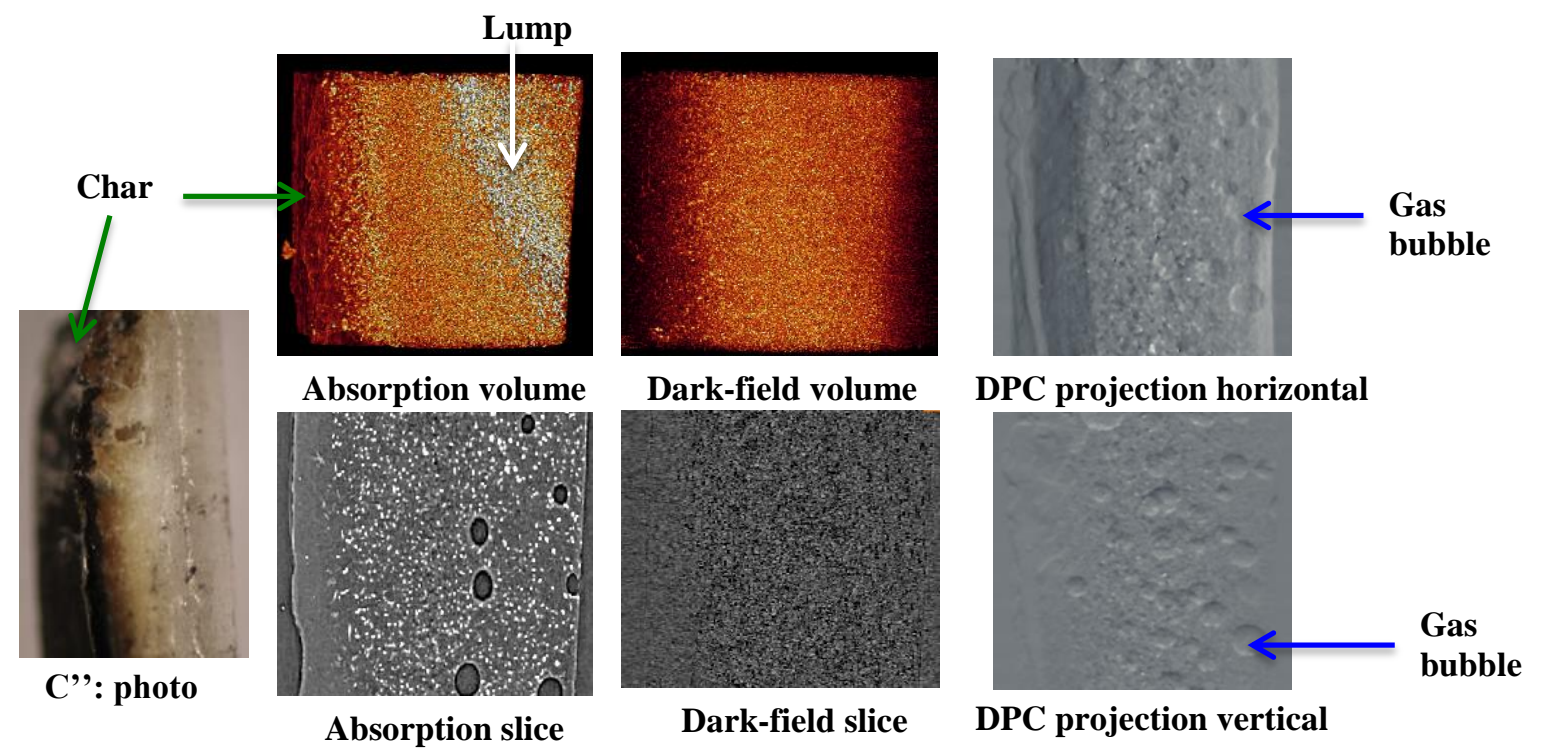

Figure S4: An optical image of sample C", having $12 \mathrm{wt} \%$ BFR and insufficient $\mathrm{Sb}_{2} \mathrm{O}_{3}$ formulation and taken from UL-94 test burn bar show a char layer and a nearly pristine interior. Tomography volumes and orthoslices of absorption, dark-field and horizontal with vertical projections of DPC show the char layer as well as an interior region that shows 
effects of heating, with possible dissolution of BFR lumps and the formation of gasbubbles.

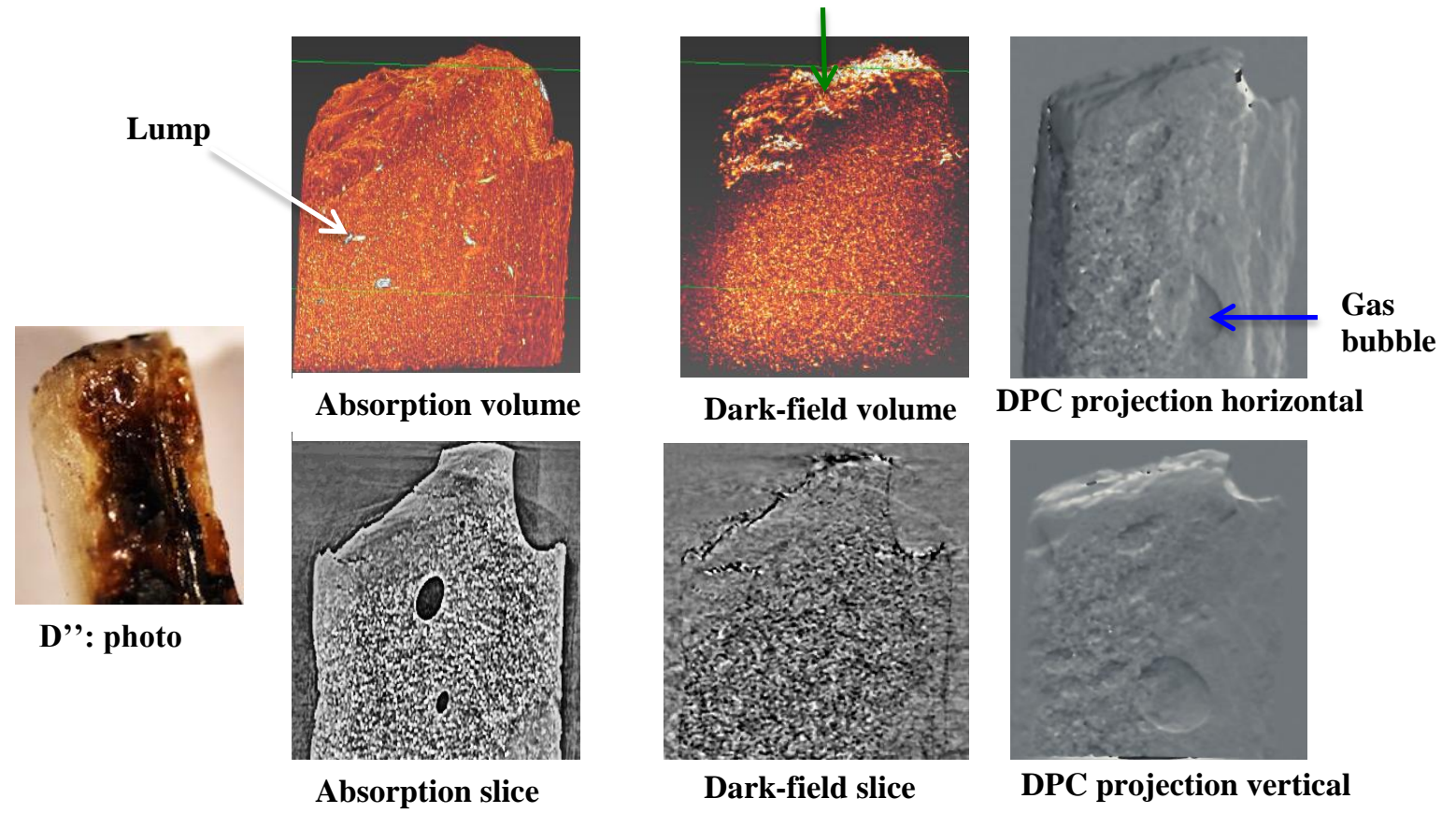

Figure S5: An optical image of sample D, having the correct BFR and $\mathrm{Sb}_{2} \mathrm{O}_{3}$ formulation and taken from UL-94 test burn bar show a char layer and a nearly pristine interior. Tomography volumes and orthoslices of absorption, dark-field and horizontal with vertical projections of DPC show the char layer as well as an interior region that shows effects of heating, especially in the dissolution of BFR lumps and the formation of gas-bubbles.
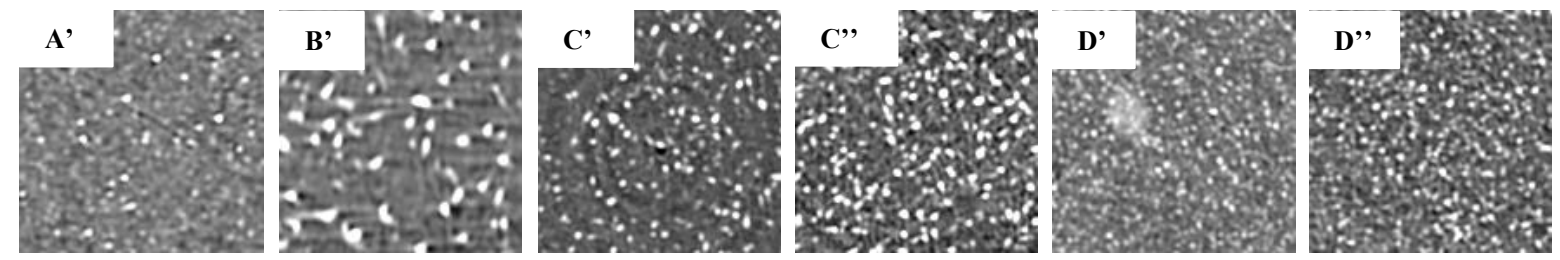

Figure S6: The autoscaling inherent in single X-ray energy imaging, as for these orthoslices from single-shot interferometry, can lead to misunderstanding of chemical distributions. Histograms of the K-edge imaging results will reveal that "lumps" are not particles of pure BFR or $\mathrm{Sb}_{2} \mathrm{O}_{3}$. 


\section{K-edge Absorption Tomography}
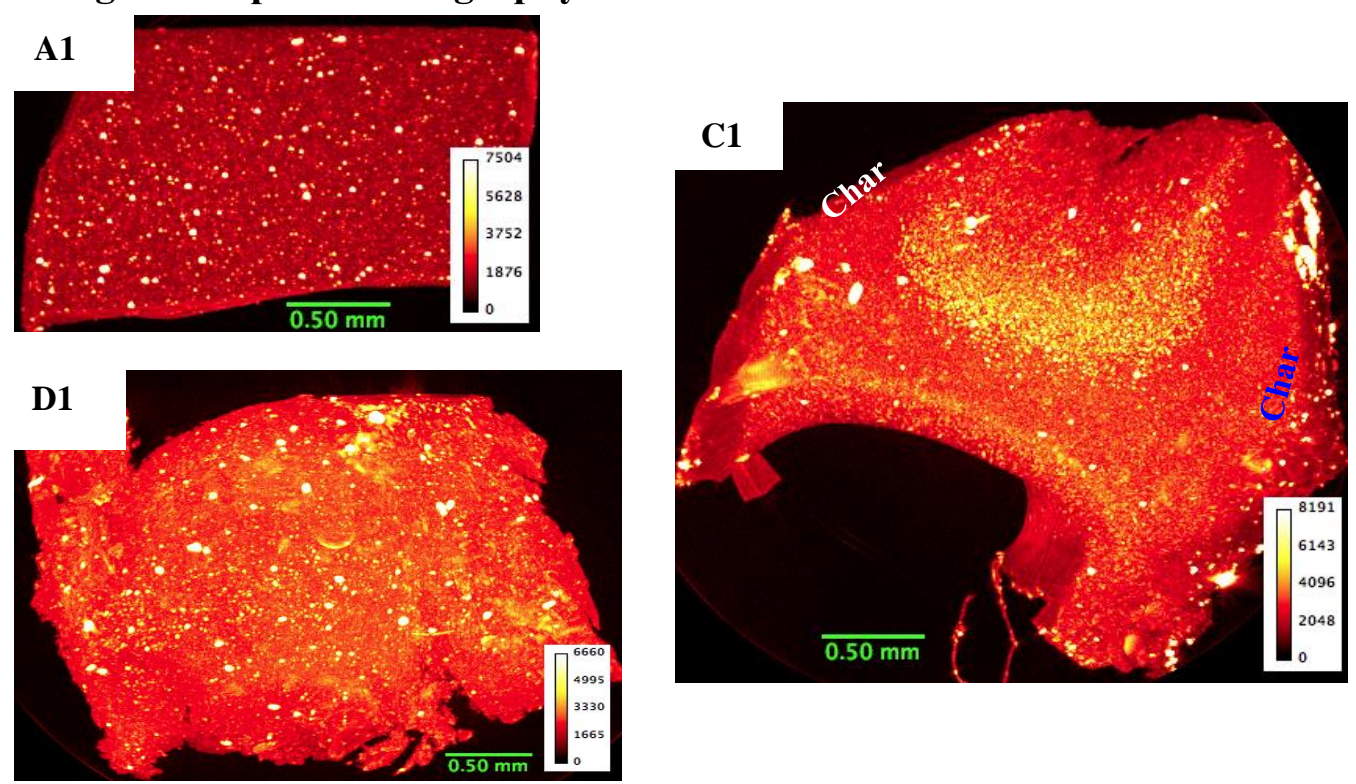

Fig S7: Stacked slice at $12 \mathrm{keV}$, below the Br edge of sample A1, C1 and D1. A "stacked slice" view (ImageJ) is based on maximum intensity along the viewing dimension. For these non-uniform shaped samples, stacked slice provides fast views for preliminary evaluation. The stacked slice view emphasizes BFR and $\mathrm{Sb}_{2} \mathrm{O}_{3}$ inhomogeneity, though gas bubbles are not seen.
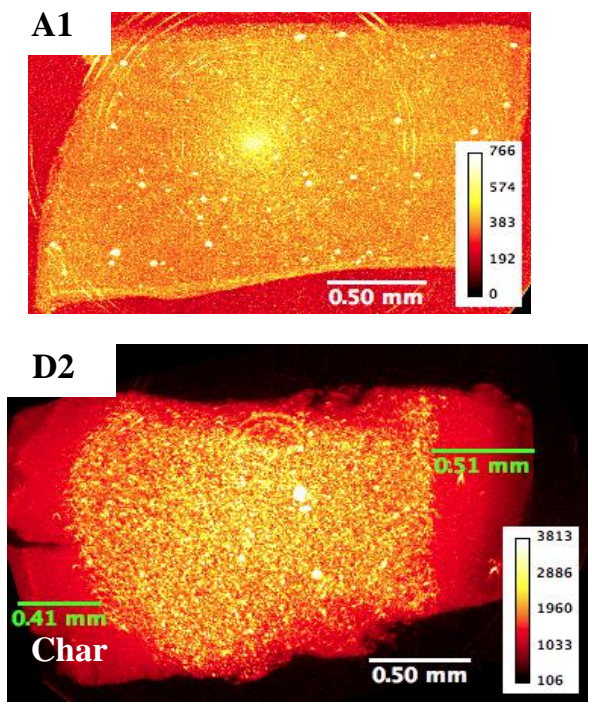
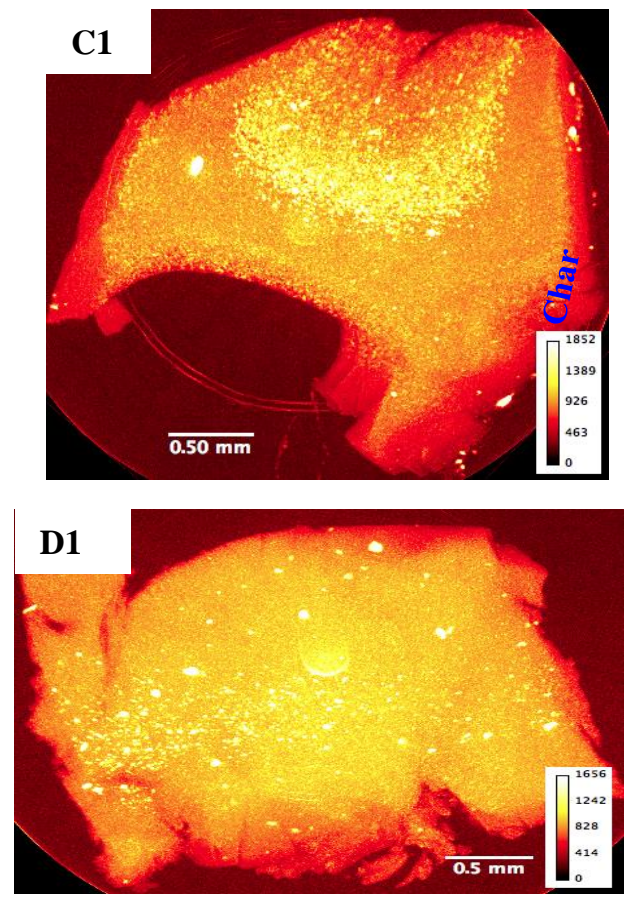
Figure S8: Stacked slice of sample A1, C1, and D1, D2 of maximum intensity at $29 \mathrm{keV}$. The sample B stacked slice is not shown since no data acquired at such X-ray energy due to absence of $\mathrm{Sb}_{2} \mathrm{O}_{3}$ in the polymer blend.

A1: $\mathrm{Sb}_{2} \mathrm{O}_{3} \mathbf{v o l} \%$

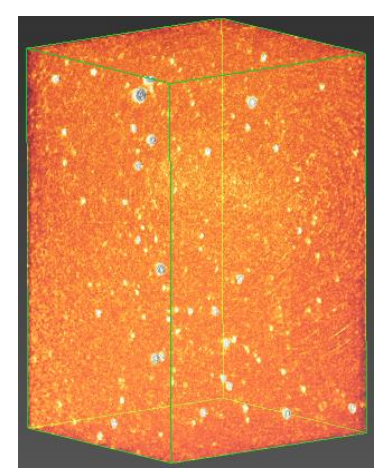

B1: BFR vol\%

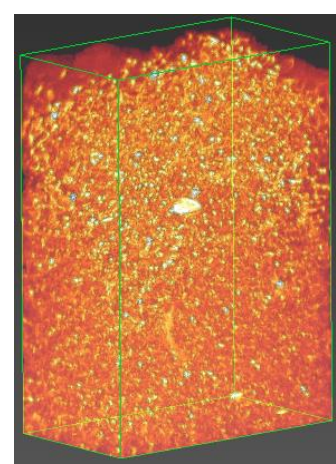

C1: BFR vol\%

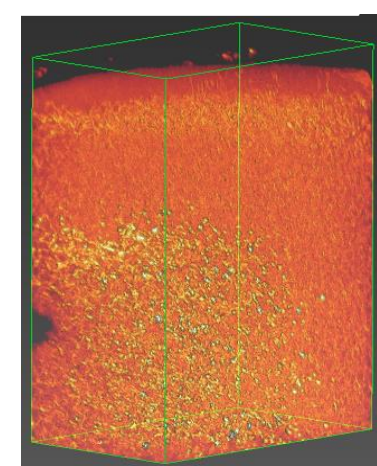

D1: BFR vol\%

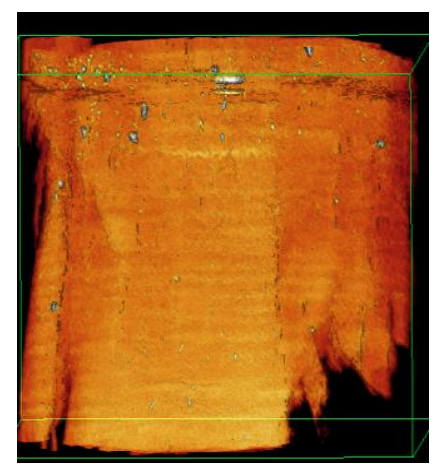

Figure S9: Volume renderings of maximum intensity of burnt samples A1, B1 and C1. These renderings are to show that BFR dissolution is added with $\mathrm{Sb}_{2} \mathrm{O}_{3}$.

Improvement in dissolution of BFR is noticed with $1 \mathrm{wt} \%$ and $4 \mathrm{wt} \%$ of $\mathrm{Sb}_{2} \mathrm{O}_{3}$ in samples $\mathrm{C}$ and D respectively when compared to sample B of only BFR $12 \mathrm{wt} \%$.

Table S1: BFR concentration of samples B1, C1, D1 and D3 with mean vol\%, standard deviation and maximum vol\% values computed from K-edge tomography.

\begin{tabular}{|cccc|}
\hline Sample & Average & Std deviation & Maximum \\
\hline B1 & 2.772 & 0.860 & 15.053 \\
\hline C1 & 3.054 & 0.802 & 17.042 \\
\hline D1 & 2.779 & 0.537 & 6.642 \\
\hline D3 & 3.711 & 0.668 & 29.058 \\
\hline
\end{tabular}




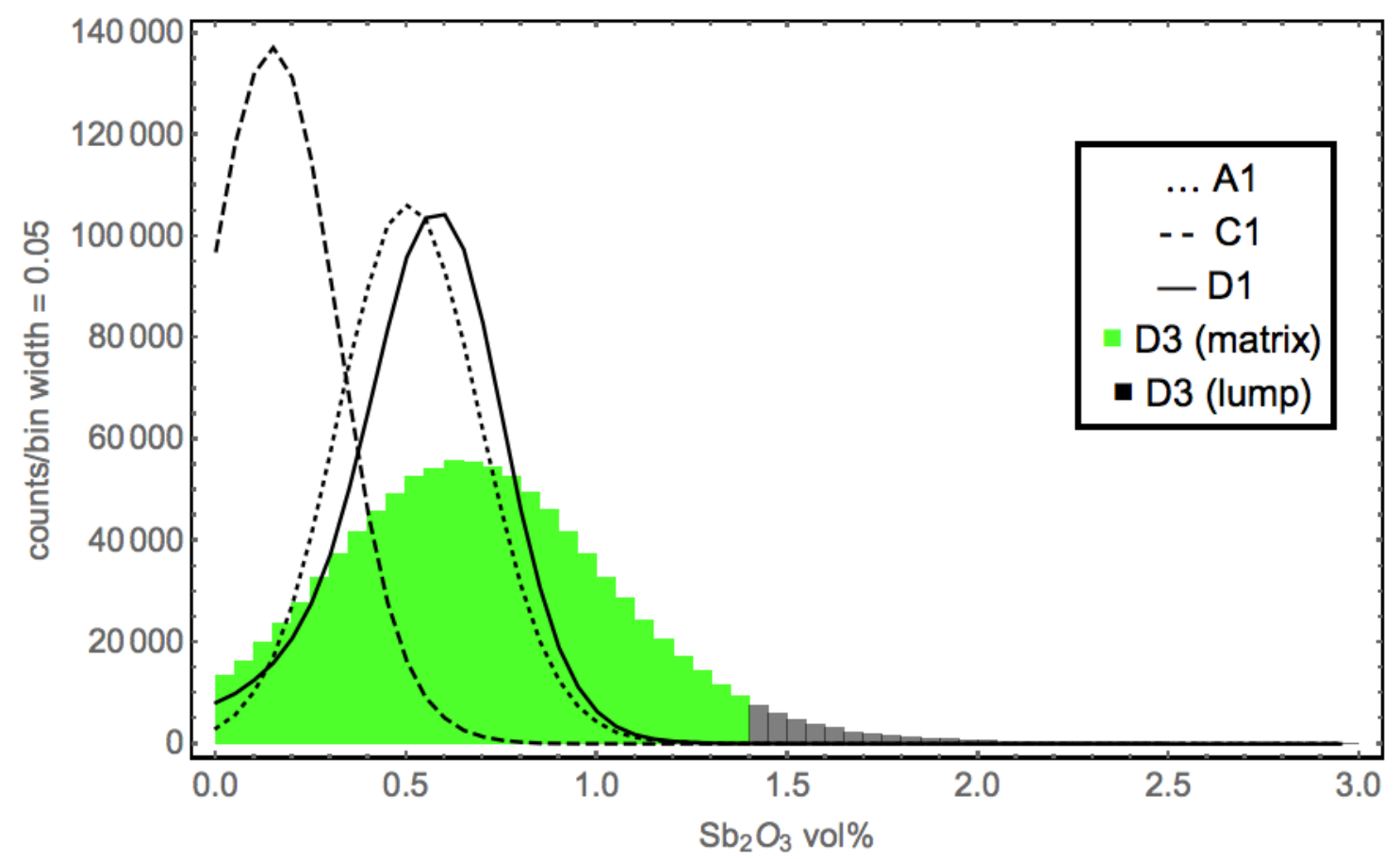

Figure S10: Histogram of $\mathrm{Sb}_{2} \mathrm{O}_{3}$ concentration in vol \% of sample A1, C1, D1 and D3 computed from K-edge tomography. The histograms are based on $10^{6}$ randomly chosen voxels within the sample. At a threshold of $1.4 \mathrm{vol} \%$, fresh/unburnt sample D3 has a fraction of 8.91 in lumps. This indicates $91 \%$ of $\mathrm{Sb}_{2} \mathrm{O}_{3}$ has dissolved in the polymer.

Table S2: $\mathrm{Sb}_{2} \mathrm{O}_{3}$ Vol\% concentration of samples A1, C1, D1 and D3 with mean, standard deviation and maximum values computed from K-edge tomography.

\begin{tabular}{|cccc|}
\hline Sample & Average & Std. deviation & Maximum \\
\hline A1 & 0.536 & 0.194 & 10.06 \\
\hline C1 & 0.222 & 0.145 & 8.70 \\
\hline D1 & 0.576 & 0.209 & 11.68 \\
\hline D3 & 0.706 & 0.386 & 14.06 \\
\hline
\end{tabular}

These figures show some of the transformation of tomography datasets of BFR and $\mathrm{Sb}_{2} \mathrm{O}_{3}$ into separate concentration vol\% of $\mathrm{BFR}$ and $\mathrm{Sb}_{2} \mathrm{O}_{3}$. These slices show burnt sample D2. 

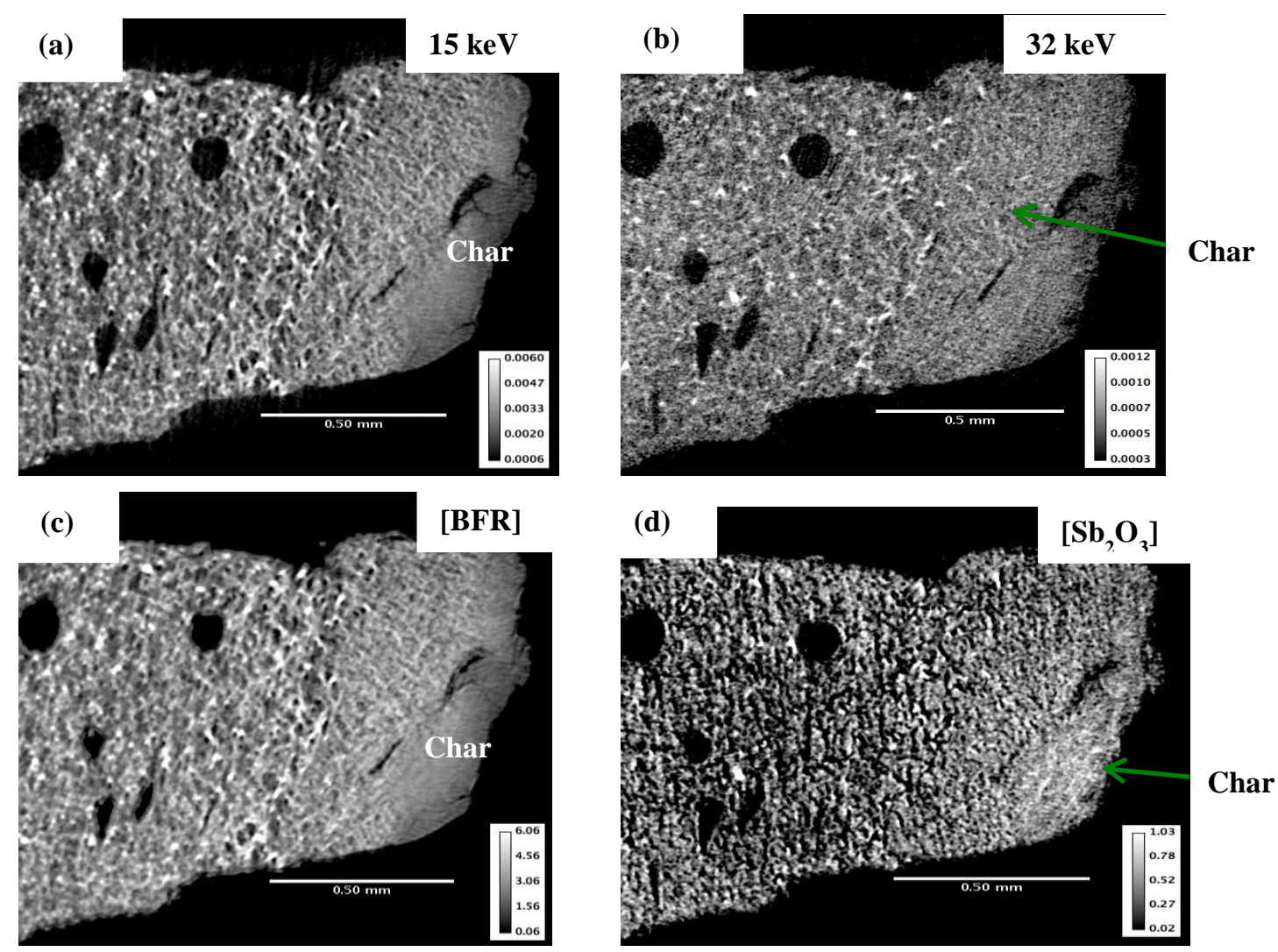

Figure S11: K-edge tomography imaging and results for burnt sample D2. (a) A representative slice of tomography at $15 \mathrm{keV}$, above the Br K-edge and (c) the fitted BFR concentration in vol\%. Areas of high X-ray absorption correspond to high BFR concentration. (b) A representative slice of tomography at $32 \mathrm{keV}$, above the Sb K-edge and (d) the fitted $\mathrm{Sb}_{2} \mathrm{O}_{3}$ concentration in vol\%. Areas of high X-ray absorption mostly correspond to high $\mathrm{Sb}_{2} \mathrm{O}_{3}$ concentration, though the $\mathrm{Br} \mathrm{X}$-ray absorption complicates the image shown in (b). 

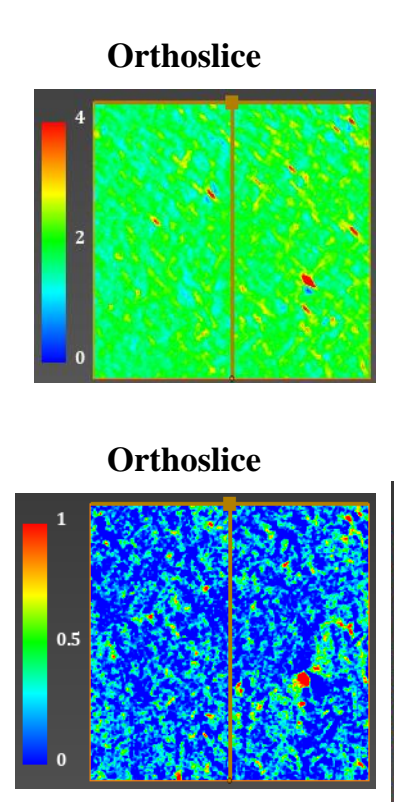

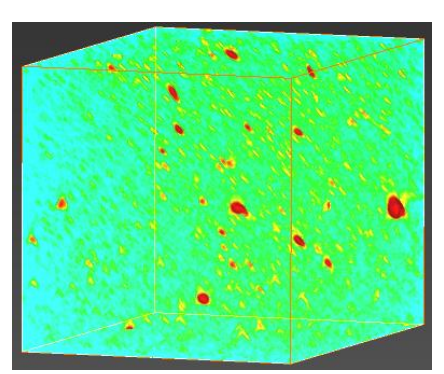

Volume rendering

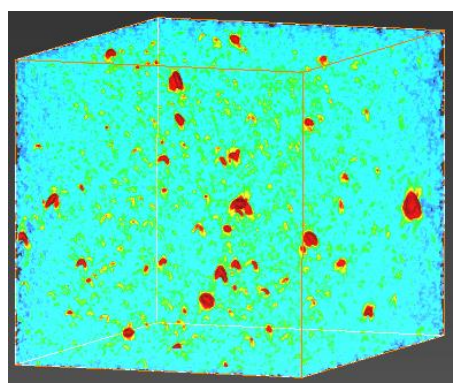

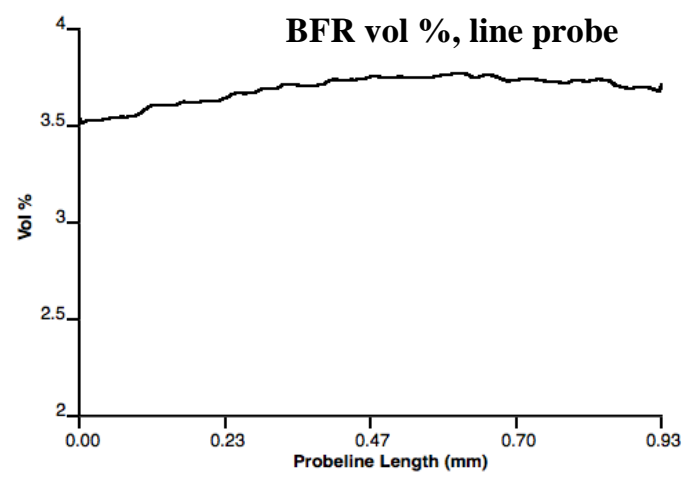

$\mathrm{Sb}_{2} \mathrm{O}_{3}$ vol \%, line probe

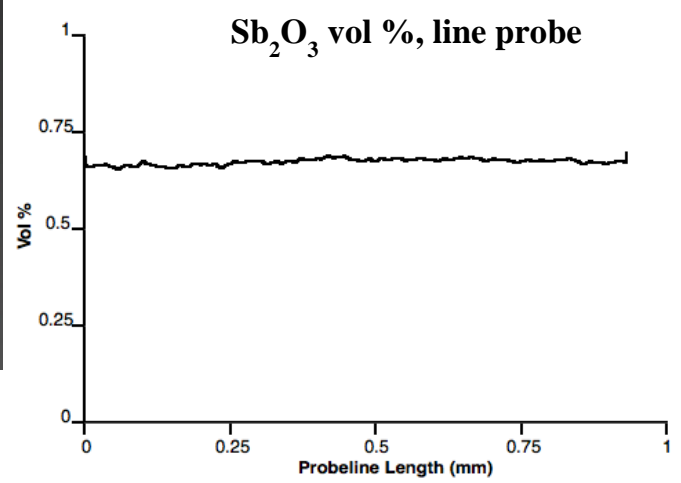

Figure S12: The orthoslice, volume rendering and line probe (radius $=10$ ) of unburnt sample D3 show the blending and good homogeneity of the flame retardant polymer sample. The histogram (Fig. 8, Table S1) shows that the voxel with maximum BFR has less than $30 \mathrm{vol} \%$ BFR. Similarly, less than $17 \mathrm{vol} \% \mathrm{Sb}_{2} \mathrm{O}_{3}$ is found in a voxel for $\mathrm{Sb}_{2} \mathrm{O}_{3}$ (Fig. $\mathrm{S} 10$, Table S2). The above figures are in accordance with histograms and maximum voxel concentrations. 
BFR

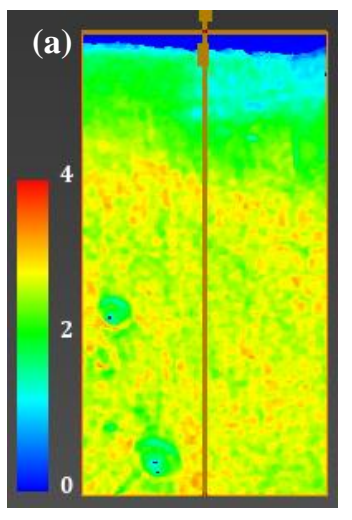

$\mathrm{Sb}_{2} \mathrm{O}_{3}$

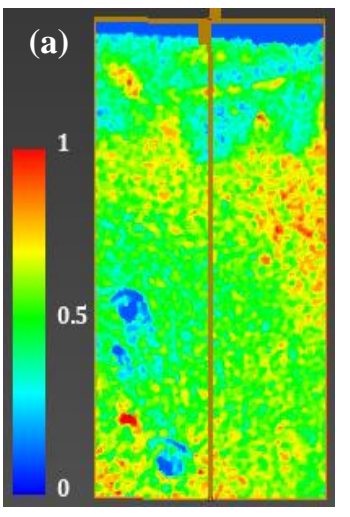

(b)

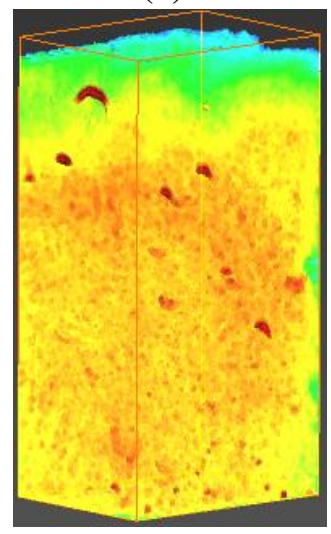

(b)

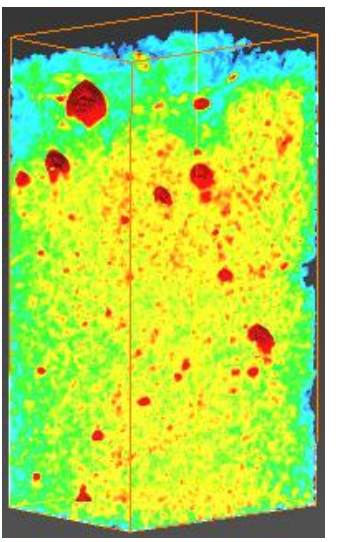

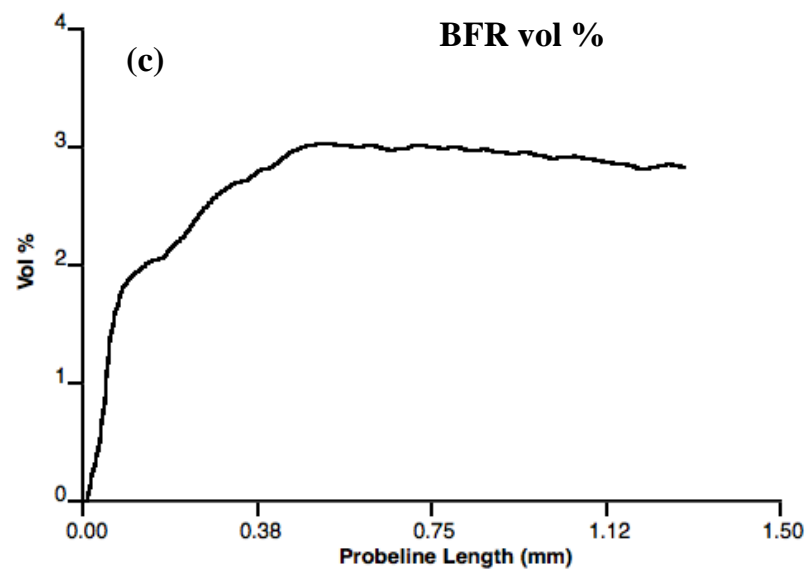

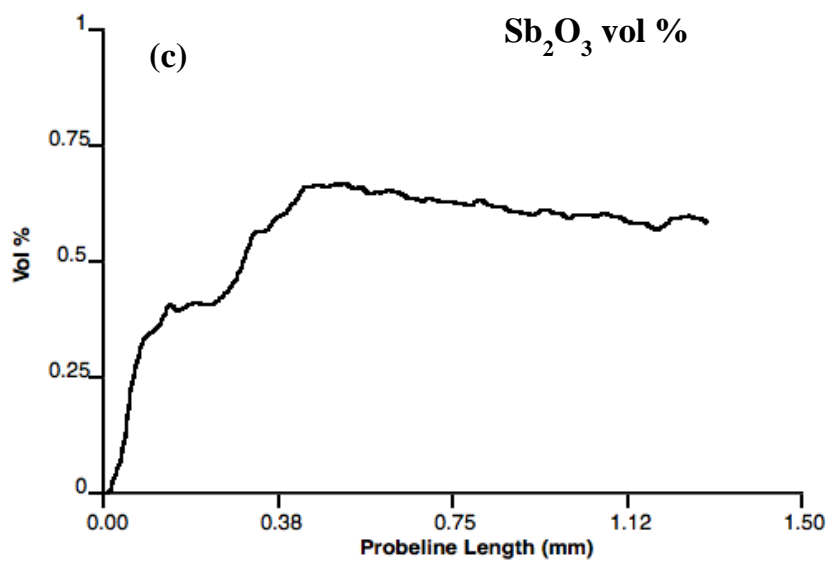

Figure S13: A 2D orthoslice (a) with a line probe (radius $=10)$ to generate graph $(\mathrm{c})$; 3D volume rendering (b) are displayed to present concentration gradient across the char layer of burnt sample D1. The line probes (c) show low concentration (vol\%) in the char layer for both BFR and $\mathrm{Sb}_{2} \mathrm{O}_{3}$. 


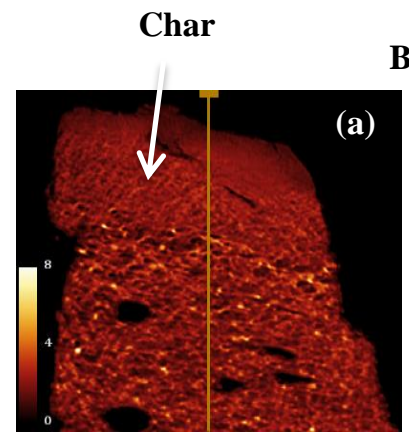

Slice; Line probe
BFR

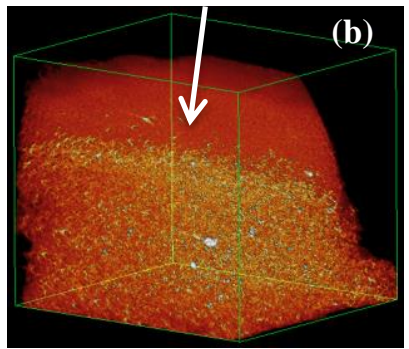

Volume rendering

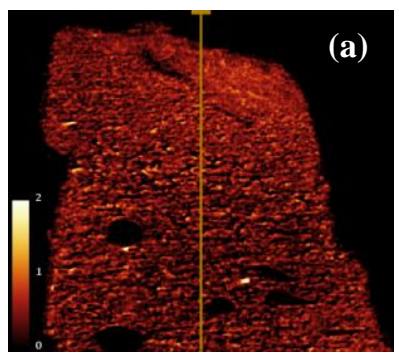

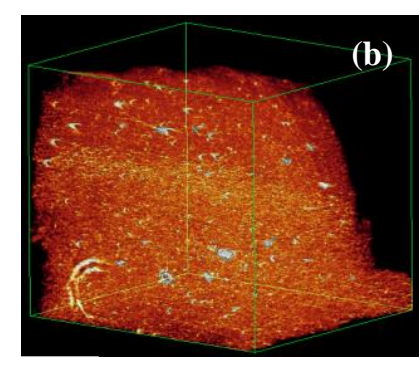

$\mathrm{Sb}_{2} \mathrm{O}_{3}$
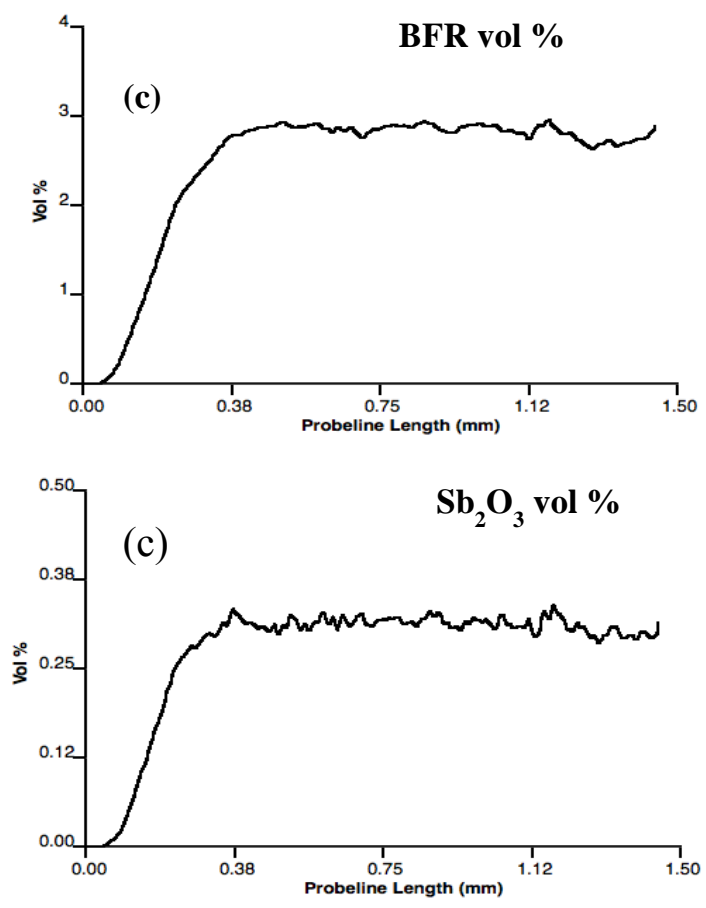

Figure S14: A 2D orthoslice (a) with a line probe (radius $=10)$ to generate graph $(\mathrm{c})$; 3D volume rendering (b) are displayed to present concentration gradient across the char layer of burnt sample D2. The line probes (c) show low concentration in the char layer for both $\mathrm{BFR}$ and $\mathrm{Sb}_{2} \mathrm{O}_{3}$.
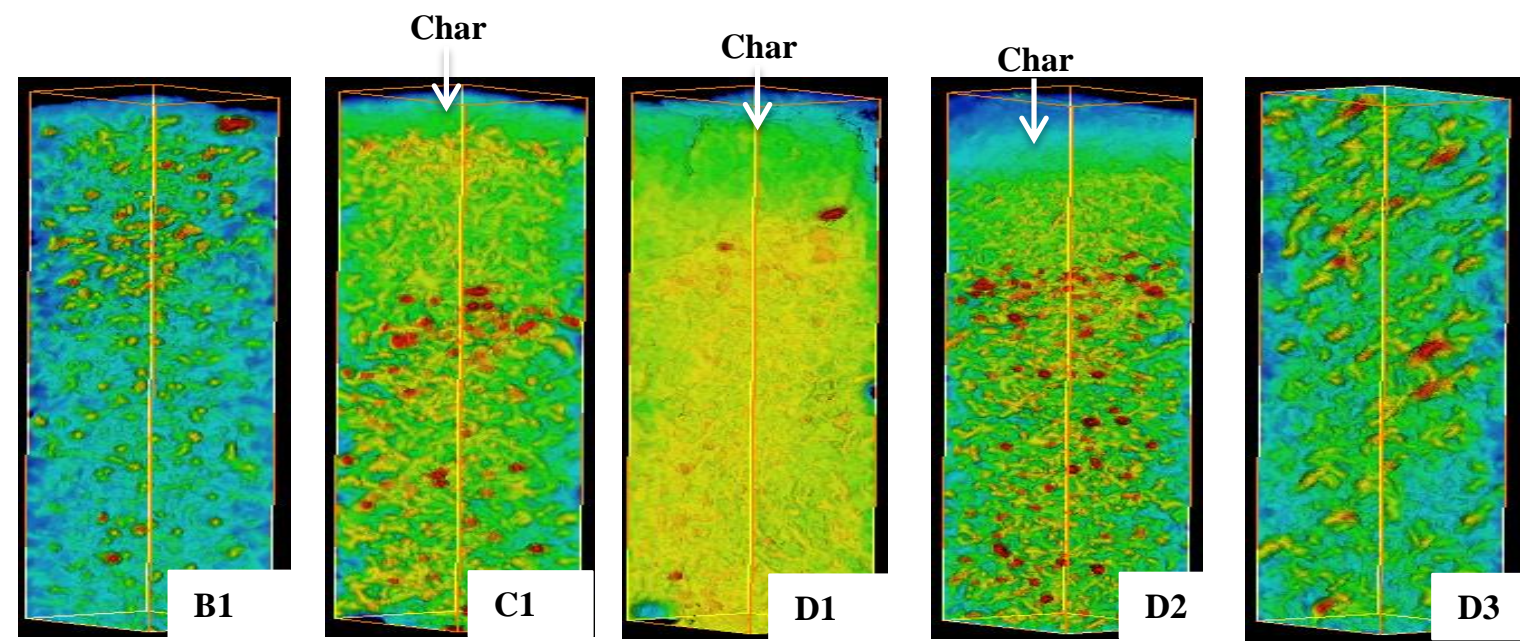

Figure S15: Volume renderings for the line probes (radius $=10$ ) for comparing BFR concentration in vol\% from the air through the char layer and into the interior for burnt B1, burnt $\mathrm{C} 1$, burnt D1, burnt D2, and pristine D3.

The X-axes (shown in Fig. S16) of the burnt samples are mutually registered based on the position of $1.5 \mathrm{vol} \%$ BFR concentration. 


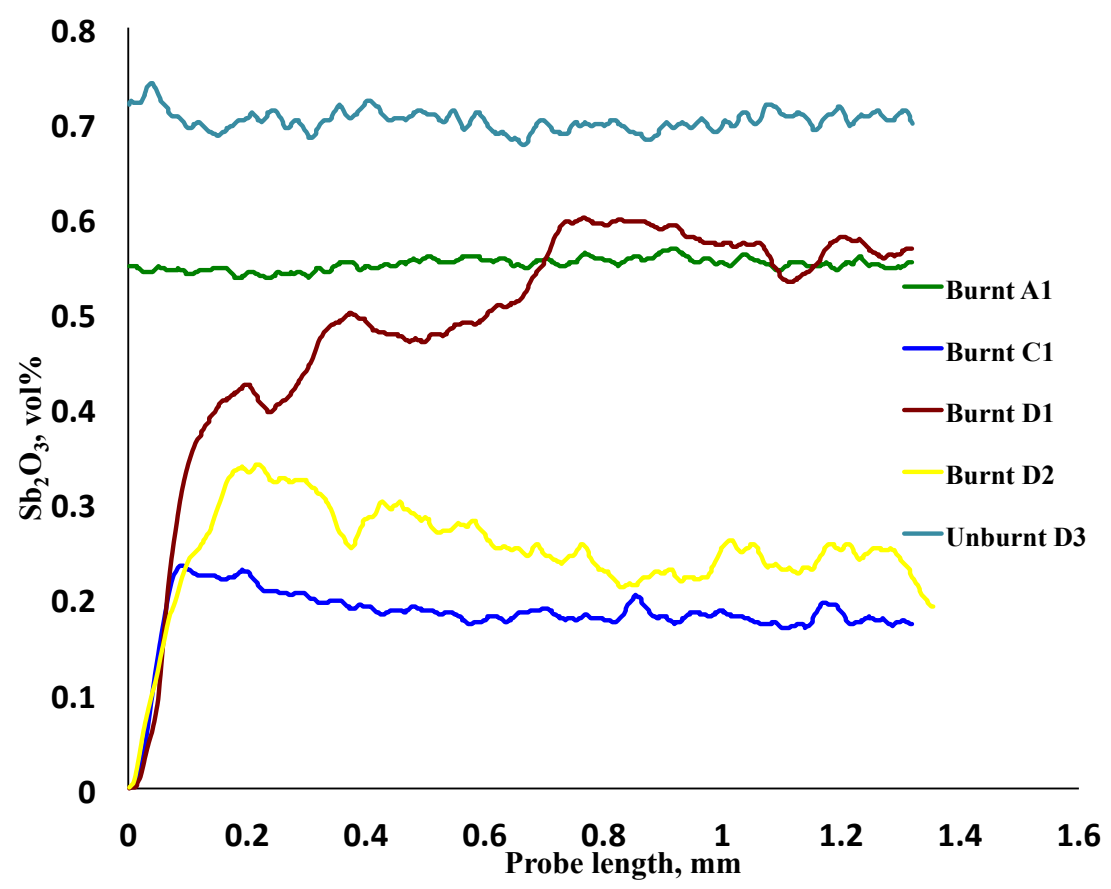

Figure S16: Line probes (radius $=10$ ) for comparing $\mathrm{Sb}_{2} \mathrm{O}_{3}$ concentration in vol\% from the air through the char layer and into the interior for burnt A1, burnt C1, burnt D1, burnt $\mathrm{D} 2$, and pristine D3. The X-axes of the burnt samples are mutually registered based on the position of $1.5 \mathrm{vol} \%$ BFR concentration. 


\section{The single-shot $\mathrm{X}$-ray grating interferometry}

The checkerboard single-shot interferometry method measures the differential phase shift along two directions. In the laboratory coordinate system, we measured DPC (vertical) and DPC (horizontal), as shown in Fig. S17. In principle, two-directional DPC data can be integrated $^{8}$ to give a phase shift image. However, phase wrap errors must be corrected before the integration. One method for phase wrap correction is based on the gradient of the absorption image ${ }^{18}$; this prior information can be useful to locate phase wraps. However, the relatively thick samples, about $2 \mathrm{~mm}$, combined with the high phase shifts of BFR and $\mathrm{Sb}_{2} \mathrm{O}_{3}$ at this low X-ray energy, $22 \mathrm{keV}$, yield too many phase wraps for this correction algorithm. The phase shifts of BFR and $\mathrm{Sb}_{2} \mathrm{O}_{3}$ are intrinsic due to the high atomic number elements, $\mathrm{Br}$ and $\mathrm{Sb}$. The phase wraps are visible in Fig $\mathrm{S} 17$ as a speckle pattern in the thick parts of the sample. The verification of DPC (vertical) and DPC (horizontal) is made easy by examination of the air-surface interface at the very bottom of the sample. In summary, higher energy X-ray imaging is needed to improve the quality of the DPC images. Thinner samples are less desirable as it is difficult to sustain a stable burn with a thin sample.
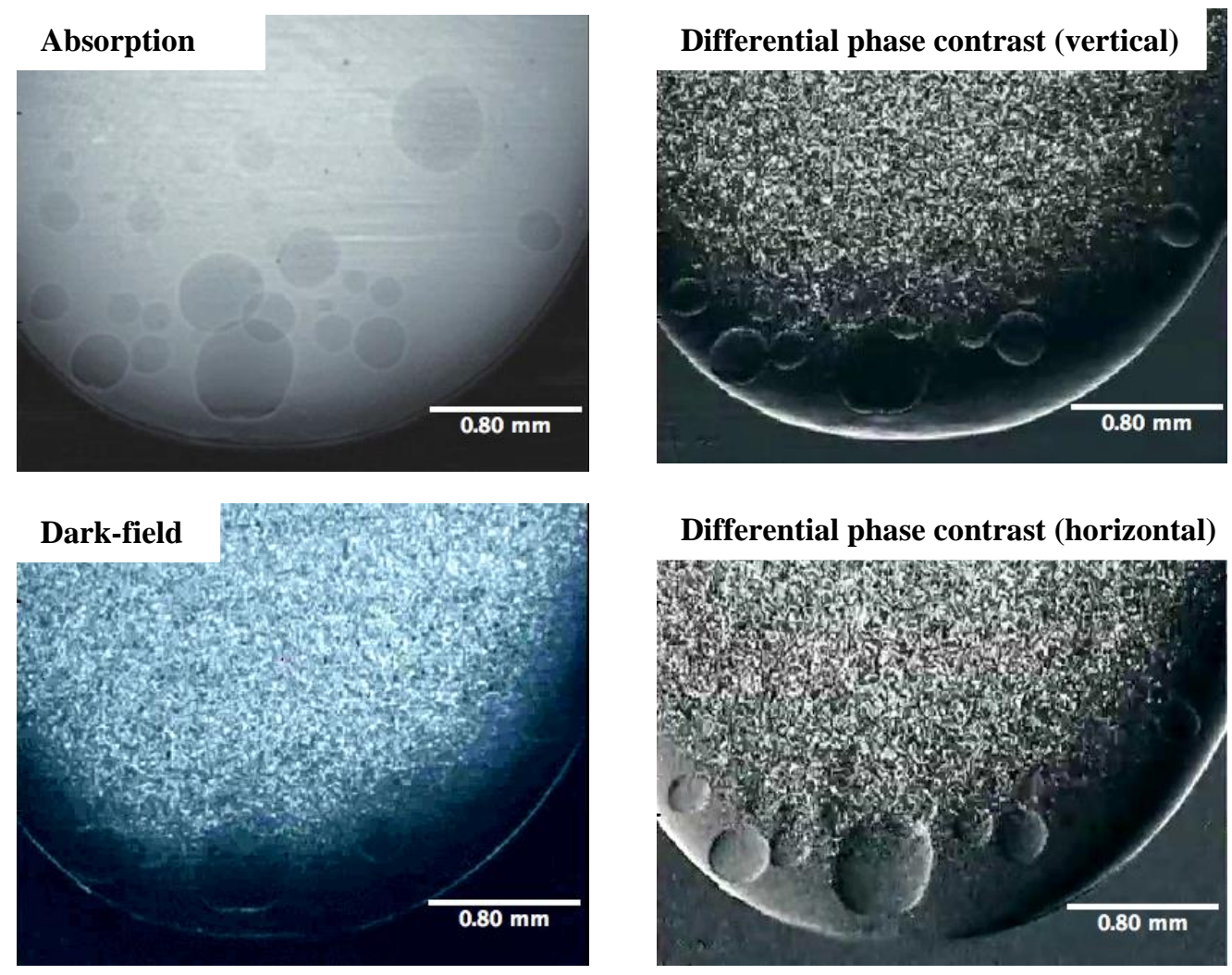

Figure S17: Phase wraps are shown in selected differential phase contrast (DPC) slices of interferometry movies, 1.3 pixel size at $22 \mathrm{keV}$. 